\title{
Maternal transmission as a symbiont sieve, and the absence of lactation in male mammals
}

\author{
George W. A. Constable, Brennen Fagan, Richard Law \\ $10^{\text {th }}$ January, 2022
}

George W.A. Constable: Department of Mathematics, University of York, York YO10 5DD, UK. Email: george.constable@york.ac.uk; ORCID ID: 0000-0001-97919571

Brennen Fagan: Leverhulme Centre for Anthropocene Biodiversity, Department of Mathematics, University of York, York YO10 5DD, UK. Email: brennen.fagan@york.ac.uk; ORCID ID: 0000-0002-8451-920X

Richard Law: York Cross-Disciplinary Centre for Systems Analysis, Ron Cooke Hub, University of York, York YO10 5GE, UK. Email: richard.law@york.ac.uk; ORCID ID: 0000-0002-5550-3567

Authors are listed alphabetically.

Gut microbiomes of humans carry a complex symbiotic assemblage of microorganisms. As in all mammals, the special mode of feeding newborn infants through milk from the mammary gland enhances the opportunity for vertical transmission of elements of this microbiome. This has potential benefits, but it also brings with it some hazards for the host. We point out here that vertical transmission from both parents would allow host populations to be invaded by elements that are deleterious. In contrast, vertical transmission, when restricted to one parent, acts as a sieve preventing the spread of such elements. Because some transmission from mother to infant is unavoidable in placental mammals, uniparental transmission of symbionts, if it were to be selected, would require some separation of the father from the newborn infant. This paper therefore puts forward the hypothesis that the asymmetry in roles of mother and father, together with the hazards that come with biparental transmission, generate a selection pressure against male lactation in humans, and in mammals in general. 
The absence of male lactation in mammals is a puzzle - there appears to be no obvious reason why it should not happen. Maynard Smith (1977) pointed out that paternal care which incorporates such feeding would be evolutionarily stable in monogamous mammals. There have been over $200 \mathrm{My}$ for male lactation to evolve (Lefèvre et al., 2010 ). Genetic control of the mammary gland is widely distributed across mammalian chromosomes (Lemay et al., 2009). Male mammals including humans have the potential to lactate (Francis et al., 1994, Reisman and Goldstein, 2018; García-Acosta et al., 2019). This requires a sufficently high level of the hormone prolactin, which is normally down-regulated in males, preventing lactation from happening (O'Hara et al. 2015). It seems there are selection pressures which keep male lactation firmly switched off.

A well-known answer to the puzzle, building on the work of Trivers (1972, 2017), is that a bias against paternal care is to be expected when there is uncertainty as to who the father is (Kokko and Jennions, 2008). The drawback to this explanation is that there are many socially monogamous mammals (Kleiman, 1977; Maynard Smith, 1977; Lukas and Clutton-Brock, 2013), and high rates of genetic monogamy amongst these species (Clutton-Brock and Isvaran, 2006). Since other forms of paternal care have evolved in such species (Kvarnemo, 2018), why has male lactation not followed suit? Daly (1979) suggested female lactation might not limit reproductive success, but this is questionable because the reproductive cycle would restart sooner if the period of female lactation was shorter (Kunz and Hosken, 2009). The levels of prolactin needed for lactation do impair male activity (Kunz and Hosken, 2009), and include loss of fertility in human males (Carani et al., 1996; Hair et al., 2002). However, this is reversible on the restoration of normal prolactin levels (Ufearo and Orisakwe, 1995). Why should a temporary reduction in male fertility not be evolutionarily sustainable in monogamous mammals? The suppression of lactation in male mammals in general, and male humans in particular remains an open question (Kunz and Hosken, 2009).

We suggest here that vertical transmission of elements of the gut microbiome, near the time at which mammals are born, provides a possible explanation. There is a basic, general problem about biparental transmission of symbionts, seen in its strongest form when a rare symbiont first colonises a host population (i.e. when most matings by carriers of the symbiont are with uninfected hosts). When transmission is biparental, a symbiont carried by the father is passed on to the offspring, just as it would be from the mother. The number of hosts with the symbiont in the next host generation is then double that under maternal transmission (Fig. 1). The problem for the host population is that this gives the symbionts a reproductive boost, enabling them to invade, even if they are harmful. Uniparental transmission provides an elegant natural solution to this problem, removing the boost and leaving the frequency of vertically transmitted symbionts unchanged when hosts reproduce.

The advantage to the host of restricting symbiont transmission to one rather than both parents can be made precise with a little algebra, previously used for investigating the evolution of anisogamy (Law and Hutson, 1992). Consider a host's symbiont community comprising a set of microbial taxa, labelled $s$. Suppose an 


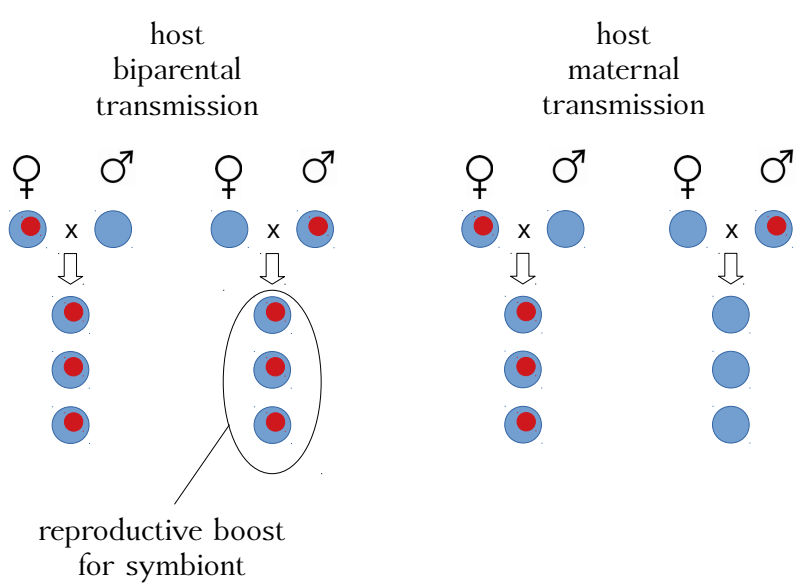

Figure 1: Biparental transmission in a host population gives a reproductive boost to symbionts. This is at its greatest when symbionts are rare in the host population, and infected hosts mate mostly with uninfected ones, as shown here. The boost is prevented by uniparental transmission, assumed here to be maternal.

additional element appears and changes the set of symbionts, the new set being labelled $S$. (We treat the new symbiont as an independent addition to a resident set of symbionts. Interactions with the resident microbiome are important (Foster et al. 2017), but would not help in understanding the simple effect of uniparental transmission.) The frequency of matings under random mating, and the symbiont sets associated with them, are as follows:

Table 1: Mating classes.

\begin{tabular}{ccccll} 
mother & $\times$ & father & frequency & $P(S)$ & $P(s)$ \\
\hline$s$ & $\times$ & $s$ & $q^{2}$ & 0 & 1 \\
$S$ & $\times$ & $s$ & $p q$ & $\alpha$ & $1-\alpha$ \\
$s$ & $\times$ & $S$ & $p q$ & $\beta$ & $1-\beta$ \\
$S$ & $\times$ & $S$ & $p^{2}$ & $\alpha+\beta-\alpha \beta$ & $(1-\alpha)(1-\beta)$
\end{tabular}

In this scheme, the frequency of symbiont set $S$ (respectively $s$ ) in the parental host generation is $p$ (respectively $q=1-p$ ). For generality, we write the probability that a female (respectively male) passes $S$ on to the next generation as $\alpha$ (respectively $\beta$ ). Thus, under biparental transmission $\alpha=1, \beta=1$, and under maternal transmission $\alpha=1, \beta=0 . P(S)$ is the probability with which the offspring of each host mating carry the symbiont set $S$, and $P(s)$ is the probability with which the offspring carry the symbiont set $s$.

The frequency of hosts carrying $S$ in the next generation, $p^{\prime}$, is given by the frequency of matings that pass $S$ on, multiplied by the fitness $w$ of hosts carrying 
$S$ relative to those that do not:

$$
p^{\prime}=\left(p q \alpha+p q \beta+p^{2}(\alpha+\beta-\alpha \beta)\right) \frac{w}{\bar{w}} .
$$

Here the mean fitness of the population, $\bar{w}$, normalises the frequencies in the next generation so that they sum to 1 . The change in frequency of hosts carrying $S$ is then

$$
\begin{aligned}
\Delta p & =p^{\prime}-p \\
& =\frac{p w}{\bar{w}}(\alpha+\beta-p \alpha \beta)-p \\
& =\frac{p}{\bar{w}}(\gamma(p+w q)-1),
\end{aligned}
$$

where $\gamma=\alpha+\beta-\alpha \beta p$.

The condition for the symbiont set $S$ to increase in the host population from one generation to the next is $\Delta p>0$. From Eq. (2), the condition is $(p+w q)>1 / \gamma$. When the new symbiont set $S$ first appears in the host population, $p \approx 0$ and $q \approx 1$, so $S$ invades if $w>1 / \gamma$. Fully biparental transmission of symbionts $(\alpha=1, \beta=1)$ implies $\gamma=2$, so $S$ invades if it gives a host fitness $w>1 / 2$ relative to $s$. This demonstrates the reproductive boost that allows it to invade even if it reduces host fitness. In contrast, fully maternal transmission of the symbionts $(\alpha=1, \beta=0)$ implies $\gamma=1$, so $S$ can only invade if it gives a host fitness $w>1$, i.e. a fitness greater than that of $s$. Symbionts with $w<1 / 2$, would not invade at all in this scheme, whether transmission is uniparental or biparental.

A similar argument could be constructed to show that transmission of symbionts through the father $(\alpha=0, \beta=1)$ rather than the mother, would be just as advantageous. However, the process of birth makes some transmission of microorganisms from the mother to infant unavoidable in placental mammals. Evidence for this includes differences observed in the gut microbiomes of infants born naturally, and those delivered by Caesarian section (Stewart et al. 2018$)$, which implies some transfer of symbionts occurs during a natural birth (Funkhouser and Bordenstein, 2013). There are also indications that bacteria can reach the uterus through the blood stream of the mother in mice (Jiménez et al., 2008), although the longstanding paradigm of the sterile womb still has support in humans (Perez-Muñoz et al. 2017; de Goffau et al., 2019). Given this basic asymmetry between the roles of mother and father in placental mammals, uniparental transmission of symbionts would be expected to operate through the mother.

In effect, maternal transmission acts as a first line of defense for hosts in the face of an unruly mob of microorganisms. It operates at the start of life as a "symbiont sieve", separating those that are beneficial to the host from those that are deleterious, and preventing the deleterious ones from spreading in the host population.

Fig. 2 illustrates the sieve in action. This shows the ultimate fate of symbionts with different effects $w$ on host fitness, under biparental and maternal transmission. 
(a) symbiont pool
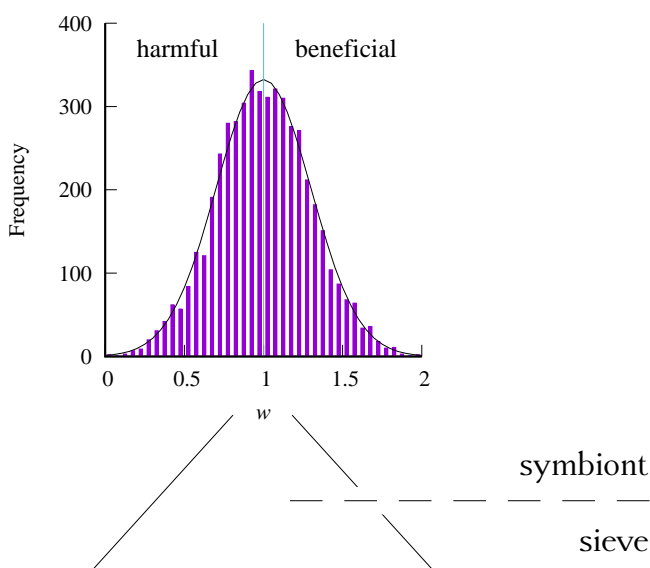

(b) biparental transmission

(c) maternal transmission
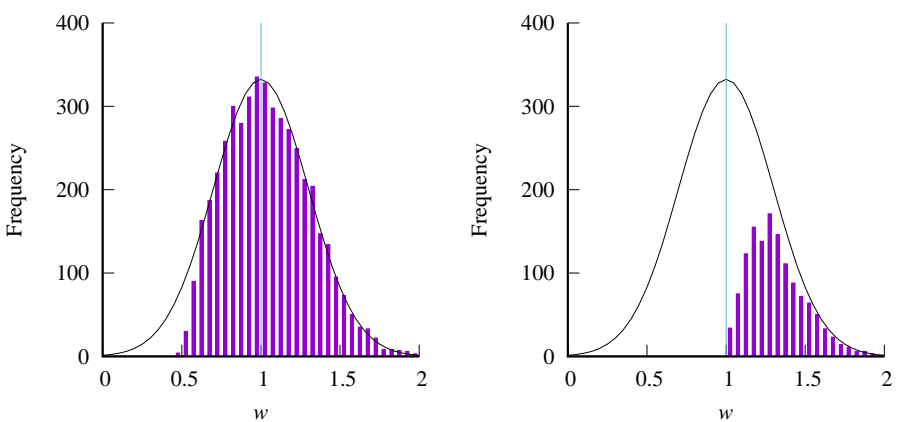

Figure 2: A numerical experiment to demonstrate maternal transmission operating as a symbiont sieve. (a) A frequency distribution from which to draw random values of $w$, the effect of symbionts on host fitness; $w<1$ : harmful; $w>1$ : beneficial. (b) Frequency distribution of $w$ in symbionts that successfully invade under biparental transmission: invasion can occur whether the symbiont is beneficial or harmful to the host. (c) Corresponding frequency distribution under maternal transmission: this sieves out harmful symbionts, only allowing invasion by symbionts beneficial to their hosts.

To do this, we constructed a continuous-time, stochastic, birth-death process with logistic density-dependence, to describe host population dynamics, controlling for vertical transmission of the symbiont (see Methods). The effect on host fitness $w$ was taken to be a random variable drawn from a normal distribution centred on $w=1$, the point of neutrality where symbionts have no effect on host fitness (Fig. 2 a). For argument's sake, the symbiont acted on the host death rate $d$ as as a factor $d / w$, so symbionts with $w>1$ were beneficial, and those with $w<1$ deleterious. We carried out 5000 independent trials of this experiment for each mode of vertical transmission. Each trial started with a host population close to the equilibrium population size (1000 hosts), 10 of which carried symbiont, and the population was tracked over time to see whether the symbiont was ultimately present in all or none of the hosts. In a total of 10000 trials, only four cases remained undecided after after 200 time units had elapsed; these were under maternal transmission with $w$ 


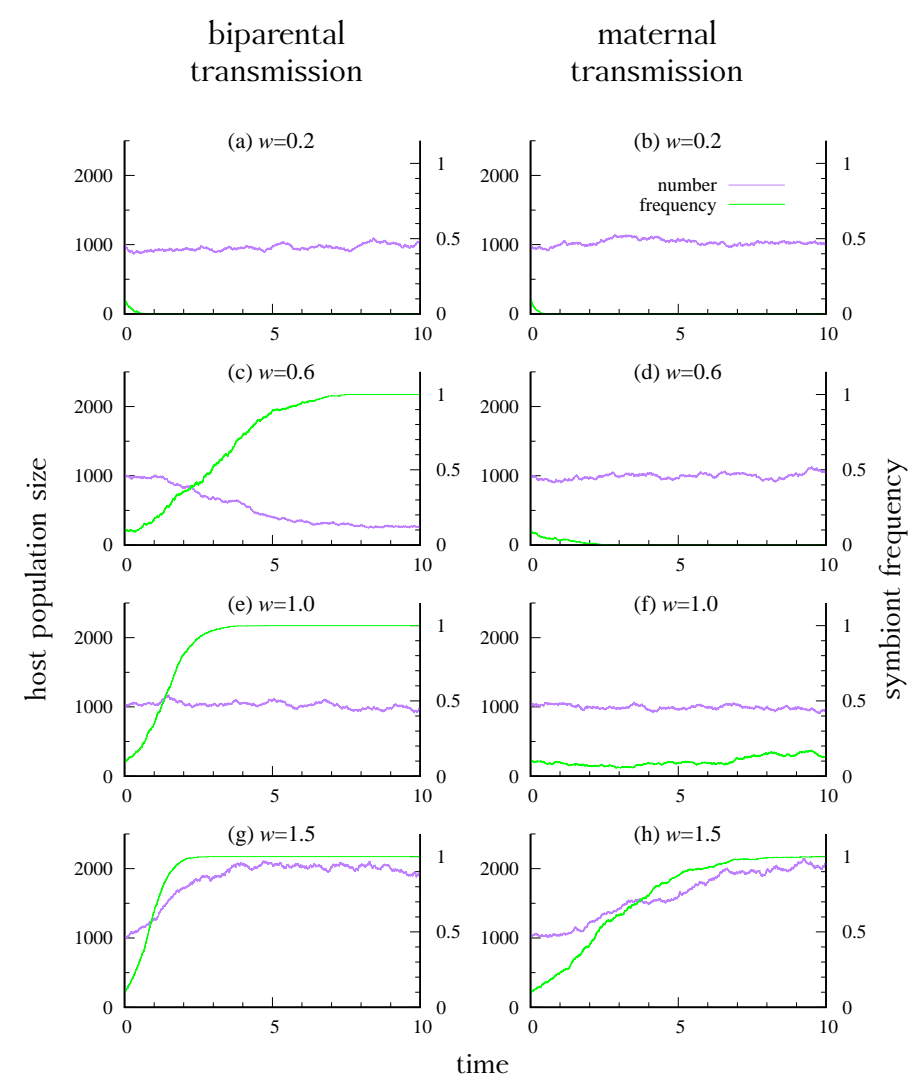

Figure 3: Example time-series of host population size, starting with 1000 individuals of which 100 carried the symbiont (i.e. a starting frequency of 0.1). In column 1 transmission of the symbiont is biparental; in column 2 it is maternal. Rows show different values of $w$ (the effect on host fitness), from the top where symbionts are harmful, to the bottom where they are beneficial. Maternal transmission protects host populations from invasion by deleterious symbionts which can occur under biparental transmission.

close to neutrality.

Fig. $2 \mathrm{~b}$ shows that the strong boost from biparental transmission allows invasion by deleterious symbionts, as long as they no more than double the death rate of hosts. In other words, the lower limit for invasion of the symbiont is $w=$ $1 / 2$, as in the simpler algebraic model above. In contrast, maternal transmission (Fig. 2c) operates as a sieve, preventing invasion by deleterious symbionts $(w<$ $1)$, while still allowing the beneficial ones to invade $(w>1)$, as in the algebraic model above. Near the point of neutrality under maternal transmission $(w=1)$, demographic stochasticity is likely to lead to extinction of the symbiont before it can get established. So the probability of invasion tends to zero, as $w \rightarrow 1$ from above.

Fig. 3 gives some examples of time series on which Fig. 2 is based, taking specific values of $w$ to show how the outcomes differ under biparental and maternal transmission. The threshold for invasion by a symbiont is $w_{0}=1 / 2$ for biparental 
and $w_{0}=1$ for maternal transmission (see Methods). Thus Fig. 3 a, b with $w=0.2$ show the symbiont being rapidly eliminated from the host population, irrespective of the mode of transmission. However, the outcomes are quite different at $w=0.6$ because the symbiont can invade under biparental but not under maternal transmission (Fig. 3c, d). At $w=1$, where the symbiont has no effect on host fitness, the reproductive boost from biparental transmission still allows rapid invasion by the symbiont, whereas its fate is the outcome of an unbiased random walk under maternal transmission (Fig. 3e, f). It is only above both invasion thresholds that the symbiont can eventually spread to all hosts (i.e. symbiont frequency $=1$ ) under both modes of transmission (Fig. 3b, h). Notice though that the protection afforded by maternal transmission comes with the cost that the symbiont spreads more slowly than it would under biparental transmission.

The prospects for taxa with biparental transmission are potentially still worse than is evident here. There are two threshold values of $w$ to consider: $w_{0}$ at which the symbiont can invade, and $w_{1}$ at which the host population size is zero at equilibrium, once the symbiont is present in all hosts (see Methods). With the parameter values used here, $w_{1}=w_{0}$ under biparental transmission. However, this depends on the biology of how the symbiont affects the host, and it would also be possible to have $w_{1}>w_{0}$. This would mean a deleterious symbiont could invade and then drive the host population to extinction. Maternal transmission prevents this lethal outcome by ensuring that only beneficial symbionts can invade.

How host genetic systems evolve and maintain maternal transmission of symbionts is a separate and interesting matter. As a deleterious symbiont is spreading under biparental transmission (Fig. 3f), it is present in some hosts and absent in others. If neither mother nor father carry the symbiont, host fitness is unaffected by the mode of transmission. The same applies if both mother and father already carry the symbiont. However, if the father carries the symbiont and the mother does not, a gene that stops transmission from the father gains an advantage, as we show in Fig. 4.

To illustrate evolution of maternal transmission, Fig. 4 uses the numerical model in the Methods, with two alternative genes $\left\{M^{+}, M^{-}\right\}$to control vertical transmission by males in the host population, during an invasion by a deleterious symbiont $(w=0.6)$. (Transmission through females is always present.) For simplicity, we assumed the genes were at a locus on the $\mathrm{Y}$ chromosome. $M^{+}$allows transmission from males, making vertical transmission biparental, whereas $M^{-}$prevents transmission from males, making vertical transmission maternal. Starting with a low frequency of $M^{-}$, a deleterious symbiont can invade the host population, because vertical transmission is predominantly biparental (Fig. 4 a). But $M^{-}$increases in frequency, because it becomes associated with hosts lacking the symbiont which are fitter than those carrying the symbiont. This association can be measured by a coefficient of disequilibrium $D$ (see Methods), which becomes positive (Fig. $4 \mathrm{~b}$ ). The association drives the host population towards maternal transmission, and eventually $M^{-}$reaches a frequency great enough to turn the tables against the symbiont. $M^{-}$goes to fixation, making vertical transmission fully maternal, and the deleteri- 
(a)

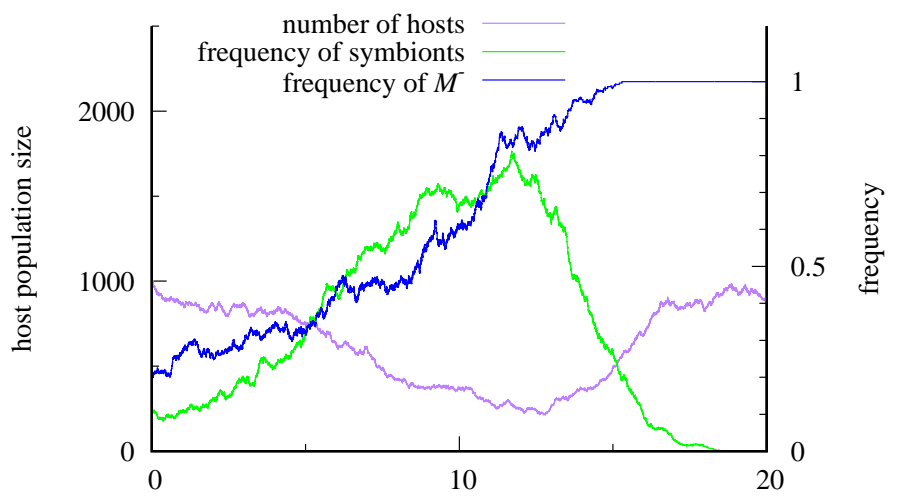

(b)

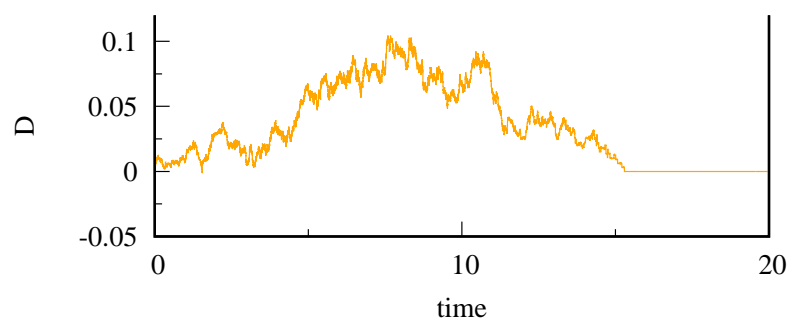

Figure 4: A harmful symbiont $(w=0.6)$ drives evolution of maternal transmission. (a) Here a host population starts mostly with biparental transmission (high frequency of host gene $M^{+}$, low frequency of $M^{-}$), and a low frequency of the symbiont in the population. This allows the symbiont to spread. The symbiont generates a selective advantage for the $M^{-}$gene which prevents male transmission. The host population then becomes dominated by maternal transmission $M^{-}$, and under these conditions the symbiont cannot persist. (b) The gene $M^{-}$ gains its advantage by becoming associated with male hosts that lack the symbiont, as measured by the coefficient of disequilibrium $D$.

ous symbiont is then eliminated from the host population. The outcome is a host population both with maternal transmission, and also with protection from this and other harmful symbionts.

This outcome would not necessarily be achieved in a single step, as it depends on initial conditions and stochastic events. So a sequence of deleterious symbionts might be required to drive the host population to a state of complete maternal transmission (i.e. frequency of gene $M^{-}=1$ ). The role of continuing immigration pressure by symbionts on the evolution of vertical transmission is an open research question for modellers. New arrivals are more likely to be harmful than beneficial to the host, but a beneficial symbiont would spread faster under biparental transmission than under maternal transmission, so there are conditions under which biparental transmission could be selected. The balance between these opposing selection pressures needs investigation - our preliminary checks suggest selection for biparental transmission is relatively weak. Maternal transmission has the advantage that it both allows invasion by beneficial symbionts, and also protects the host population from harmful symbionts, which biparental transmission cannot do. An- 
other matter for future research are the consequences of partial controls on vertical transmission (i.e. values of $\alpha$ and $\beta$ other than 0 or 1 ), which could be quite feasible in open systems like gut microbiomes.

There are many ways in which hosts can limit vertical transmission of microorganisms from the father, through physiology, behaviour and social structure. Human breast milk nicely illustrates of one of them. Breast milk contains a large microbiome (Hunt et al., 2011; Stinson et al., 2020). This becomes a major feature in the composition of the infant's gut microbiome (Stewart et al., 2018), and there is little doubt that some vertical transmission of the microbiome is taking place. The source of the microbiome is a subject of current research. But at least some elements appear to come from the maternal gut, possibly reaching the breast through an entero-mammary pathway (Oikonomou et al., 2020), and are transmitted from mother to infant via breast milk (Jost et al., 2014). As shown above, deleterious elements would spread through host populations if both parents took part in breast feeding. In practice, the hormone prolactin is down-regulated in males to a level low enough to stop lactation. This has the immediate effect of greatly restricting the contribution fathers can make to the gut microbiome of newborn infants.

We cannot prove that the danger of invasion by deleterious elements in the gut microbiome is the reason why there is no male lactation. So this argument is put forward as a hypothesis. However, milk production for feeding infants is a defining property of mammals and lactation by males is almost invariably switched off in them. The symbiont sieve thus offers a novel solution to this longstanding puzzle, and points to an important mechanism by which mammals exert some control over the composition of their gut microbiomes during transmission of microorganisms from one generation to the next.

The hypothesis offers a new perspective on several lines of research. First, it is testable. Widespread deleterious symbionts propagating solely through vertical transmission in breast milk of humans and other mammals would be inconsistent with the hypothesis. This is not to claim that there are no maternally transmitted pathogens, which is clearly incorrect, but such symbionts would need additional mechanisms to spread, such as direct horizontal infection from host to host. Secondly it leads to the question as to what beneficial roles are played by maternally transmitted symbionts in gut microbiomes. These are open systems colonized within host generations by a diverse set of microorganisms, and of remarkable complexity (Foster et al., 2017). The order in which these systems are assembled leaves a lasting impact on the structure of the microbiome in mice (Martínez et al., 2018), suggesting a key role for those present near the time of birth. For example, Bifidobacterium, which occurs in breast milk, is known to inhibit the growth pathogenic bacteria and aid digestion of the milk Arboleya et al., 2016)

Thirdly, mammalian gut microbiomes are notable for the way in which their structure mirrors the phylogenetic relationships of their mammalian hosts (phylosymbiosis), notwithstanding their species richness. This is thought to be related to special traits of mammals including viviparous birth, milk production and parental 
care (Mallott and Amato, 2021). The role of physiological filters by hosts on their symbionts is recognised in this, but not the basic property of maternal transmission in separating beneficial from harmful symbionts. It could be that positive frequencydependent selection between beneficial symbionts and their hosts acts to conserve microbiome structure, contributing to the signal of host phylogeny. Fourthly are the exceptions. The Dayak fruit bat is one of the very few mammals in which males with functional mammary glands have been documented under natural conditions (Francis et al. 1994). Gut microbiomes of bats are unlike those of other mammals, being quite unpredictable (as in birds), and lacking clear relationships with host phylogeny (Song et al., 2020). This could be in part a consequence of adaptations for flight, including lower body mass, a reduction gut size and shorter retention times of materials in the gut. This leads to the possibility that dependence on the gut microbiome is low enough to release the Dayak fruit bat from some selection pressures for maternal transmission, allowing both parents to contribute to feeding (Maynard Smith, 1977).

Lastly, we note that maternal transmission of symbionts is a widespread phenomenon in the natural world (Funkhouser and Bordenstein, 2013). It is possible that maternal transmission has evolved on a number of occasions as a simple mechanism that has the effect of sieving beneficial from harmful symbionts.

\section{Acknowledgments}

We thank the University of York Reading Group on Stability and Complexity in which discussions on the dynamics of gut microbiomes led to this wok, and C. M. Law and J. W. Pitchford for comments on the the paper.

\section{Methods}

To investigate the dynamics of a host population with vertically transmitted symbionts, we constructed a continuous-time, stochastic, birth-death process with logistic density-dependence. This is more nuanced than the algebraic model in the text, the density dependence allowing a wider range of outcomes, including extinction of the host driven by deleterious symbionts. The state of the host population at time $t$ consisted of the sex $\left\{o, \sigma^{\prime}\right\}$ and the presence or absence $\{+,-\}$ of an added symbiont in each host individual $i=1, \ldots, n(t)$, i.e.:

$$
\begin{array}{lll}
i_{\odot}^{+}: & \text {female } & \text { with symbiont set } S \\
i_{\sigma^{+}}^{+}: & \text {male } & \text { with symbiont set } S \\
i_{\odot}^{-}: & \text {female } & \text { with symbiont set } s \\
i_{\sigma^{*}}^{-}: & \text {male } & \text { with symbiont set } s .
\end{array}
$$


We write $n^{+}(t)$ as the number of hosts with set $S$, and $n^{-}(t)$ with set $s$. Thus $n^{+}(t)$ gives a measure of the abundance of the additional symbiont in the host population at time $t$, and $n^{+}(t) / n(t)$ is its frequency in the host population.

The probability per unit time of death $d_{i}$ of host $i$ contained a logistic component common to all individuals, and a further dependence on symbiont status:

$$
\begin{array}{ll}
i_{\circ}^{+}: & d_{i}=\left(d_{0}+d^{\prime} n(t)\right) / w \\
i_{\sigma^{\prime}}^{+}: & d_{i}=\left(d_{0}+d^{\prime} n(t)\right) / w \\
i_{\text {甲 }}^{-}: & d_{i}=d_{0}+d^{\prime} n(t) \\
i_{\sigma^{+}}^{-}: & d_{i}=d_{0}+d^{\prime} n(t),
\end{array}
$$

where $d_{0}$ is an intrinsic death rate and $d^{\prime}$ scales the density-dependent component of death. The death rate was modified by a factor $1 / w$ in hosts carrying the additional symbiont (symbiont set $S$ ). This describes the effect of the symbiont on its host: $w=1$ is neutral, a beneficial symbiont $(w>1)$ lowers $d_{i}$ by a factor $1 / w$, and a deleterious symbiont $(0<w<1)$ raises $d_{i}$ by a factor $1 / w$.

The per-female probability per unit time of giving birth $b_{0}$ was set to be independent of host population density. The sex of the newborn individual was assigned with an equal probability 0.5 to be female or male. Thus the probability per unit time with which a mother gave birth to a daughter, the key measure for host population growth, was $b_{0} / 2$. Whether the symbiont was present $(+)$ or absent $(-)$ in a newborn host individual depended on the mode of vertical transmission:

$$
\begin{array}{lll}
\text { biparental: } & + & \text { if mother }+, \text { or if father }+ \\
& - & \text { if mother }-, \text { and if father }- \\
\text { maternal: } & + & \text { if mother }+ \\
& - & \text { if mother }-
\end{array}
$$

Mating was assumed to be at random, so a random father was chosen from the males present in the population for biparental transmission. This completes the specification of the stochastic, birth-death process.

We carried out realisations of the stochastic process using the Gillespie algorithm (Gillespie, 1977). Numerical results were obtained with parameter values: $b_{0}=4$, $d_{0}=1, d^{\prime}=0.001$. The computations for Fig. 2 used a random value of $w$ drawn from a normal distribution with mean 1, and standard deviation 0.3 . The initial number of host individuals was 1000, the equilibrium point in the absence of the symbiont, and the symbiont was introduced to 10 individuals at the start, randomly distributed between females and males. Realisations were terminated when the symbionts were present in all hosts, or absent in all hosts, or the run-time had reached 200 time units. 5000 independent realisations of each mode of vertical transmission were carried out. In almost all instances the outcome was presence in all hosts, or absence in all hosts. The four exceptions out of 10000 realisations were under maternal transmission, with $w$ close to neutrality.

We extended the stochastic process to describe evolution of vertical transmission using two alternative genes at a locus on the $\mathrm{Y}$ chromosome. Male hosts were 
classified according to the gene they carried, $M^{+}$switching on male transmission, and $M^{-}$switching off male transmission. Symbiont transmission from females was present throughout, so vertical transmission was biparental in crosses with $\mathrm{M}^{+}$ males and maternal in crosses with $M^{-}$males. There are four classes of males depending on transmission gene $\left\{M^{+}, M^{-}\right\}$and symbiont status $\{+,-\}$. To measure the association between transmission gene and symbiont status, we write the frequency of the classes in males as

$$
\begin{array}{lll}
p_{1}: & \text { frequency of } i_{M^{-}}^{-} \\
p_{2}: & \text { frequency of } i_{M^{-}}^{+} \\
p_{3}: & \text { frequency of } i_{M^{+}}^{-} \\
p_{4}: & \text { frequency of } i_{M^{+}}^{+},
\end{array}
$$

where $p_{1}+p_{2}+p_{3}+p_{4}=1$. The coefficient of disequilibrium, $D=p_{1} p_{4}-p_{2} p_{3}$, then measures the association between transmission gene and symbiont status. $D$ is positive if symbionts are under-represented in $M^{-}$males and over-represented in $M^{+}$ males and negative if vice versa. This completes the specification of the stochastic, birth-death process with evolution of vertical transmission. Computation of Fig. 4 was carried out with parameter values set to be the same as those in Figs 2 and 3.

We note that a system of ordinary differential equations can be constructed for the mean behaviour of the stochastic process using the associated master equation. We have used this to check the results of the stochastic realisations (results not shown), and to gain further understanding of the dynamics. In the absence of evolution (Figs 2, 3), the associated equations are

$$
\begin{aligned}
\frac{\mathrm{d} x^{+}}{\mathrm{d} t} & =\frac{b_{0}}{2} \frac{1}{x^{+}+x^{-}}\left[\left(x^{+}\right)^{2}(\alpha+\beta-\alpha \beta)+(\alpha+\beta) x^{-} x^{+}\right] \\
& -x^{+}\left[\frac{d_{0}+2 d^{\prime} V\left(x^{+}+x^{-}\right)}{w}\right] \\
\frac{\mathrm{d} x^{-}}{\mathrm{d} t} & =\frac{b_{0}}{2} \frac{1}{x^{+}+x^{-}}\left[\left(x^{-}\right)^{2}+(2-\alpha-\beta) x^{-} x^{+}+(1-\alpha)(1-\beta)\left(x^{+}\right)^{2}\right] \\
& -x^{-}\left[d_{0}+2 d^{\prime} V\left(x^{+}+x^{-}\right)\right]
\end{aligned}
$$

assuming a 1:1 sex ratio to reduce the dimensionality of the system from four to two. The state variables are the density of female hosts $x^{+}$with the symbiont set $S$, and the density $x^{-}$with the symbiont set $s$. These state variables come from dividing the number of females with and without the additional symbiont $\left(n^{+}, n^{-}\right)$ by system size $V\left(x^{+}=n^{+} / V, x^{-}=n^{-} / V\right)$. The mating classes are as defined in Table 1, the proportion of females (respectively males) passing the symbiont set $S$ on to the next host generation being $\alpha$ (respectively $\beta$ ).

We used the differential equations to describe the dynamics under biparental transmission (setting $\alpha=1, \beta=1$ ), and under maternal transmission (setting $\alpha=1, \beta=0)$. The additional symbiont was added close to a boundary equilibrium 
point $I$ of the host population

$$
\begin{aligned}
& \hat{x}_{I}^{+}=0 \\
& \hat{x}_{I}^{-}=\frac{1}{2 d^{\prime} V}\left(\frac{b_{0}}{2}-d_{0}\right) .
\end{aligned}
$$

The initial per-capita rate of increase of hosts carrying the symbiont at this equilibrium point is

$$
\begin{aligned}
\text { biparental }: & \left.\frac{1}{x^{+}} \frac{\mathrm{d} x^{+}}{\mathrm{d} t}\right|_{I}=b_{0}\left(1-\frac{1}{2 w}\right) \\
\text { maternal }: & \left.\frac{1}{x^{+}} \frac{\mathrm{d} x^{+}}{\mathrm{d} t}\right|_{I}=\frac{b_{0}}{2}\left(1-\frac{1}{w}\right),
\end{aligned}
$$

giving a threshold fitness $w_{0}$ above which invasion of the symbiont happens as $w_{0}=1 / 2$ for biparental transmission, and $w_{0}=1$ for maternal transmission. $\mathrm{A}$ second equilibrium point $I I$ occurs at

$$
\begin{aligned}
\hat{x}_{I I}^{+} & =\frac{1}{2 d^{\prime} V}\left(\frac{w b_{0}}{2}-d_{0}\right) \\
\hat{x}_{I I}^{-} & =0,
\end{aligned}
$$

where every host carries the symbiont. This is unaffected by the mode of transmission, but, unless the symbiont is neutral $(w=1)$, the symbiont changes the equilibrium host population size when it is present in all hosts. There is a threshold fitness $w_{1}$ at which the symbiont causes extinction of the host population $\left(\hat{x}_{I I}^{+}=0\right)$ at $w_{1}=2 d_{0} / b_{0}$.

With the model, parameter values, and initial conditions used here, the thresholds for invasion by the symbiont $w_{0}$ and extinction of the host population $w_{1}$ are $w_{0}=w_{1}=0.5$ under biparental transmission, and $w_{0}=1, w_{1}=0.5$ under maternal transmission. Thus maternal transmission protects the host population from extinction, but biparental transmission could bring the host population size close to zero. We have found other model implementations in which $w_{0}<w_{1}$ under biparental transmission, which implies such transmission allows invasion by symbionts that definitely lead to host extinction. 


\section{REFERENCES}

Arboleya, S., Stanton, C., Ryan, C. A., Dempsey, E., and Ross, P. R. (2016). Bosom buddies: the symbiotic relationship between infants and bifidobacterium longum ssp. longum and ssp. infantis. genetic and probiotic features. Annual review of food science and technology, 7:1-21.

Carani, C., Granata, A. R. M., Fustini, M. F., and Marrama, P. (1996). Prolactin and testosterone: their role in male sexual function. International Journal of Andrology, 19(1):48-54.

Clutton-Brock, T. H. and Isvaran, K. (2006). Paternity loss in contrasting mammalian societies. Biology Letters, 2(4):513-516.

Daly, M. (1979). Why don't male mammals lactate? Journal of theoretical Biology, 78:325-345.

de Goffau, M. C., Lager, S., Sovio, U., Francesca Gaccioli, F., Cook, E., Peacock, S. J., Parkhill, J., Charnock-Jones, D. S., and S, S. G. C. (2019). Human placenta has no microbiome but can contain potential pathogens. Nature, 572:329-334. doi:/10.1038/s41586-019-1451-5.

Foster, K. R., Schluter, J., Coyte, K. Z., and Rakoff-Nahoum, S. (2017). The evolution of the host microbiome as an ecosystem on a leash. Nature, 548(7665):43-51.

Francis, C. M., Anthony, E. L. P., Brunton, J. A., and Kunz, T. H. (1994). Lactation in male fruit bats. Nature, 367:691-692.

Funkhouser, L. J. and Bordenstein, S. R. (2013). Mom knows best: the universality of maternal microbial transmission. PLoS Biol, 11:e1001631. doi:10.1371/journal.pbio.1001631.

García-Acosta, J., San Juan-Valdivia, R., A.D., F.-M., Lorenzo-Rocha, N., and Castro-Peraza, M. (2019). Trans* pregnancy and lactation: A literature review from a nursing perspective. Int J Environ Res Public Health., 17(1):44.

Gillespie, D. T. (1977). Exact stochastic simulation of coupled chemical reactions. The journal of Physical Chemistry, 81:2340-2361.

Hair, W., Gubbay, O., Jabbour, H., and Lincoln, G. (2002). Prolactin receptor expression in human testis and accessory tissues: localization and function. Molecular human reproduction, 8(7):606-611.

Hunt, K. M., Foster, J. A., Forney, L. J., Schütte, U. M. E., Beck, D. L., Abdo, Z., Fox, L. K., Williams, J. E., McGuire, M. K., and McGuire, M. A. (2011). Characterization of the diversity and temporal stability of bacterial communities in human milk. PLoS ONE, 6:e21313. doi:10.1371/journal.pone.0021313.

Jiménez, E., Mariń, M. L., Martiń, R., Odriozola, J. M., Olivares, M., Xaus, J., Fernández, L., and Rodríguez, J. M. (2008). Is meconium from 
healthy newborns actually sterile? Research in Microbiology, 159:187-193. doi:10.1016/j.resmic.2007.12.007.

Jost, T., Lacroix, C., Braegger, C. P., Rochat, F., and Chassard, C. (2014). Vertical mother-neonate transfer of maternal gut bacteria via breastfeeding. Environmental Microbiology, 16:2891-2904. doi:10.1111/1462-2920.12238.

Kleiman, D. G. (1977). Monogamy in mammals. The Quarterly review of biology, $52(1): 39-69$.

Kokko, H. and Jennions, M. D. (2008). Parental investment, sexual selection and sex ratios. Journal of evolutionary biology, 21(4):919-948.

Kunz, T. H. and Hosken, D. J. (2009). Male lactation: why, why not and is it care? Trends in Ecology 83 Evolution, 24(2):80-85.

Kvarnemo, C. (2018). Why do some animals mate with one partner rather than many? a review of causes and consequences of monogamy. Biological Reviews, 93(4):1795-1812.

Law, R. and Hutson, V. (1992). Intracellular symbionts and the evolution of uniparental cytoplasmic inheritance. Proceedings of the Royal Society of London Series B, 248:69-77.

Lefèvre, C. M., Sharp, J. A., and Nicholas, K. R. (2010). Evolution of lactation: Ancient origin and extreme adaptations of the lactation system. Annual Review of Genomics and Human Genetics, 11:219-238. doi:10.1146/annurevgenom-082509-141806.

Lemay, D., Lynn, D., and Martin, W. e. a. (2009). The bovine lactation genome: insights into the evolution of mammalian milk. Genome Biol., 10:R43.

Lukas, D. and Clutton-Brock, T. H. (2013). The evolution of social monogamy in mammals. Science, 341(6145):526-530.

Mallott, E. K. and Amato, K. R. (2021). Host specificity of the gut microbiome. Nature Reviews Microbiology, 19:639-653. doi:10.1038/s41579-021-00562-3.

Martínez, I., Maldonado-Gomez, M. X., Gomes-Neto, J. C., Kittana, H., Ding, H., Schmaltz, R., Joglekar, P., Cardona, R. J., Marsteller, N. L., Kembel, S. W., et al. (2018). Experimental evaluation of the importance of colonization history in early-life gut microbiota assembly. Elife, 7:e36521.

Maynard Smith, J. (1977). Parental investment: a prospective analysis. Animal Behaviour, 25:1-9.

Oikonomou, G., Addis, M. F., Chassard, C., Nader-Macias, M. E. F., Grant, I., Delbès, C., Bogni, C. I., Le Loir, Y., and Even, S. (2020). Milk microbiota: What are we exactly talking about? Frontiers in Microbiology, 11:60:doi:10.3389/fmicb.2020.00060. 
O’Hara, L., Curley, M., Tedim Ferreira, M., Cruickshanks, L., Milne, L., and Smith, L. B. (2015). Pituitary androgen receptor signalling regulates prolactin but not gonadotrophins in the male mouse. PLOS ONE, 10(3):1-18.

Perez-Muñoz, M. E., Arrieta, M.-C., Ramer-Tait, A. E., and Walter, J. (2017). A critical assessment of the "sterile womb" and "in utero colonization" hypotheses: implications for research on the pioneer infant microbiome. Microbiome, 5:48. doi:10.1186/s40168-017-0268-4.

Reisman, T. and Goldstein, Z. (2018). Case report: Induced lactation in a transgender woman. Transgender Health, 3:24-26. doi:10.1089/trgh.2017.0044.

Song, S. J., Sanders, J. G., Delsuc, F., Metcalf, J., Amato, K., Taylor, M. W., Mazel, F., Lutz, H. L., Winker, K., Graves, G. R., Humphrey, G., Gilbert, J. A., Hackett, S. J., White, K. P., Skeen, H. R., Kurtis, S. M., Withrow, J., Braile, T., Miller, M., McCracken, K. G., Maley, J. M., Ezenwa, V. O., Williams, A., Blanton, J. M., McKenzie, V. J., and Knight, R. (2020). Comparative analyses of vertebrate gut microbiomes reveal convergence between birds and bats. mBio, 11:e02901-19. doi:10.1128/mBio.02901-19.

Stewart, C. J., Ajami, N. J., O'Brien, J. L., Hutchinson, D. S., Smith, D. P., Wong, M. C., Ross, M. C., Lloyd, R. E., Doddapaneni, H. V., Metcalf, G. A., Muzny, D., Gibbs, R. A., Vatanen, T., Huttenhower, C., Xavier, R. J., Rewers, M., Hagopian, W., Toppari, J., Ziegler, A.-G., She, J.-X., Akolkar, B., Lernmark, A., Hyoty, H., Vehik, K., Krischer, J. P., and Petrosino, J. F. (2018). Temporal development of the gut microbiome in early childhood from the teddy study. Nature, 562:583-588. doi:10.1038/s41586-018-0617-x.

Stinson, L. F., Sindi, A. S. M., Cheema, A. S., Lai, C. T., Mühlhäusler, B. S., Wlodek, M. E., Payne, M. S., and Geddes, D. T. (2020). The human milk microbiome: who, what, when, where, why, and how? Nutrition Reviews, 79:529-543. doi:10.1093/nutrit/nuaa029.

Trivers, R. L. (1972). Parental investment and sexual selection. In Campbell, B., editor, Sexual Selection and the Descent of Man, page 136-179. Aldine Publishing, Chicago.

Trivers, R. L. (2017). Parental investment and sexual selection. Routledge.

Ufearo, C. and Orisakwe, O. (1995). Restoration of normal sperm characteristics in hypoprolactinemic infertile men treated with metoclopramide and exogenous human prolactin. Clinical Pharmacology 85 Therapeutics, 58(3):354-359. 